\title{
CONSERVAÇÃO PÓS-COLHEITA DE GUAVIRA (Campomanesia sp.) ${ }^{1}$
}

\author{
RAQUEL PIRES CAMPOS 2 , PRISCILA AIKO HIANE ${ }^{3}$, MARIA ISABEL LIMA RAMOS 3 , \\ MANOEL MENDES RAMOS FILHO ${ }^{3}$, MARIA LIGIA RODRIGUES MACEDO
}

RESUMO - Este trabalho foi realizado com o objetivo de avaliar a conservação pós-colheita de guavira (Campomanesia sp.), em função da utilização de 1-MCP e de atmosfera modificada passiva, além do armazenamento à temperatura ambiente e a $11^{\circ} \mathrm{C}$. Os frutos foram colhidos no ponto de maturidade fisiológica, subdivididos em parcelas e submetidos à aplicação de 1-Metilciclopropeno (1-MCP) (700 $\eta L . L^{-1} / 12$ horas) e à atmosfera modificada passiva por meio do uso de embalagem plástica. Foram realizadas análises de perda de massa, sólidos solúveis, acidez titulável, vitamina $C$, fenóis totais, atividade antioxidante e aparência geral dos frutos. O uso de 1-MCP não reduziu a perda de massa dos frutos, tanto em armazenamento ambiente quanto refrigerado, o que foi verificado com o uso da atmosfera modificada passiva. $\mathrm{O}$ armazenamento refrigerado reduziu a perda de massa dos frutos e prolongou a vida útil destes com manutenção dos teores de vitamina $\mathrm{C}$, fenóis totais e atividade antioxidante. Os teores finais de sólidos solúveis e vitamina $\mathrm{C}$ dos frutos não apresentaram interferência significativa em relação aos tratamentos utilizados, e os valores de acidez titulável aumentaram ao final do período de armazenamento, com exceção dos frutos tratados com 1-MCP associado com atmosfera modificada e armazenados em ambiente. O uso de 1-MCP e de atmosfera modificada passiva prolongaram a vida útil dos frutos tanto em armazenamento ambiente, quanto refrigerado, com melhor aparência geral dos frutos, principalmente quando associados.

Termos para indexação: atmosfera modificada, compostos bioativos, armazenamento refrigerado, 1-MCP.

\section{POST-HARVEST CONSERVATION OF GUAVIRA (Campomanesia sp.)}

\begin{abstract}
This study aimed to evaluate the postharvest conservation of guavira (Campomanesia sp.), depending on the use of 1-MCP and modified atmosphere, as well as storage at room temperature and at $11^{\circ} \mathrm{C}$. The fruits were harvested at physiological maturity, divided into portions and treated with 1- methylcyclopropene (1-MCP) ( $700 \eta L . L^{-1}$ for 12 hours) and modified atmosphere, with plastic packaging. The fruits were submitted to the analysis of weight loss, soluble solids, titratable acidity, vitamin C, total phenols and antioxidant activity and general appearance. The use of 1-MCP did not reduce the fruit weight loss in both ambient and cold storage, which was verified using the modified atmosphere. The cold storage reduced the mass loss of the fruits and extended the shelf life maintaining the levels of vitamin $\mathrm{C}$, total phenolics and antioxidant activity contents. The final concentration of soluble solids and vitamin $\mathrm{C}$ of the fruits showed no significant interference in relation to treatments. The titratable acidity values increased during the storage period, with the exception of fruits treated with 1-MCP associated with modified atmosphere and stored in room temperature. The use of 1-MCP and modified atmosphere extended the shelf life of fruits both in storage environment and cold, with a better overall appearance of the fruit, especially when combined.
\end{abstract}

Index terms: modified atmosphere, bioactive compounds, cold storage, 1-MCP.

\footnotetext{
1(Trabalho 180-11). Recebido em: 06-06-2011. Aceito para publicação em: 04-01-2012.

${ }^{2}$ Dr. Pesquisador DCR em Tecnologia pós-colheita (Fundect/CNPq/UFMS). e-mail: raquel.campos@ufms.br

${ }^{3}$ Prof. Dr. do Dept ${ }^{\circ}$. de Tecnologia de Alimentos e Saúde Pública, Universidade Federal de Mato Grosso do Sul (UFMS), Caixa Postal 549, CEP 79070-900, Campo Grande-MS. E-mails: priscila.hiane@ufms.br; maria-isabel.ramos@ufms.br; manoel.filho@ufms.br.
} 


\section{INTRODUÇÃO}

Os frutos nativos do Cerrado apresentam sabores especiais, com elevados teores de açúcares, proteínas, vitaminas, minerais e fibras, possuindo grande aceitação popular. A utilização de tecnologias pós-colheita pode viabilizar o desenvolvimento sustentável da agricultura familiar e de pequenas comunidades rurais, através do aumento do período de comercialização dos frutos, com melhor aproveitamento da produção e agregação de valor, além de incentivar o consumo de alimentos regionais com potencial valor nutritivo e funcional.

Perspectivas promissoras para exploração de frutos tropicais não tradicionais se devem aos níveis consideráveis de vitamina $\mathrm{C}$, antocianinas, carotenoides e compostos fenólicos, além da capacidade antioxidante destes frutos (RUFINO et al., 2010). Muita atenção tem sido focada para a atividade antioxidante total presente em frutas e hortaliças, devido aos diversos constituintes presentes que possuem propriedades de reduzir o nível do estresse oxidativo (HASSIMOTO et al., 2005), e consequentemente diminuir o risco de diversas doenças cardivasculares, neurodegenerativas e câncer (HALLIWELL, 2007; KAUR;KAPOOR, 2002).

A guavira ou gabiroba, pertencente à família Myrtaceae e ao gênero Campomanesia, é de grande abundância na região do Cerrado. As folhas e os frutos deste gênero possuem algumas propriedades medicinais com ação anti-inflamatória, antidiarreica e antisséptica das vias urinárias, utilizada contra reumatismo e como desobstruente do fígado (RODRIGUES; CARVALHO, 2001). Apresenta baixo teor energético, devido à reduzida concentração de macronutrientes, especialmente lipídios $(0,12$ g 100 $\mathrm{g}^{-1}$ ), e contém bons conteúdos de cálcio, zinco, ferro e fibras (SILVA et al., 2008). Os frutos da espécie C. adamantinum são suculentos, ácidos e levemente adocicados, com potencial para serem utilizados in natura, na indústria de alimentos e como flavorizantes na indústria de bebidas (VALLILO et al., 2006).

Assim como outras espécies de pequenos frutos, a guavira apresenta baixa conservação pós-colheita e ainda não são adotadas tecnologias adequadas para a manutenção da vida útil com qualidade. A refrigeração é o método mais econômico para o armazenamento prolongado de frutos frescos, sendo a temperatura o fator ambiental mais importante por controlar todos os processos fisiológicos e bioquímicos associados à senescência (CHITARRA; CHITARRA, 2005). O armazenamento refrigerado proporcionou maior período de conservação póscolheita em caqui (BRACKMANN et al., 2003) e em goiaba 'Kumagai' (MORGADO et al., 2010) e menor perda de qualidade em manga (DOLLHOJO et al., 2009).

O uso da atmosfera modificada, empregando filmes plásticos, limita as trocas gasosas e a perda de água dos frutos para o ambiente, reduzindo o metabolismo do produto e prolongando sua vida pós-colheita (CHITARRA;CHITARRA, 2005). É prático e pouco oneroso, podendo aumentar o período de oferta de frutos com qualidade (STEFFENS et al., 2009). O 1-metilciclopropeno (1-MCP) é um regulador vegetal, inibidor competitivo do etileno, retardando o amadurecimento de frutos (CHITARRA; CHITARRA, 2005). Resultados positivos foram obtidos em goiaba serrana (AMARANTE et al., 2008), em goiabas 'Kumagai' (CERQUEIRA et al., 2009), em jambo (PLAINSIRICHAI et al., 2010) e em mangaba (CAMPOS et al., 2011). Este trabalho foi realizado com o objetivo de avaliar a vida útil e as características físicas, químicas e sensoriais de guaviras, submetidas ou não à refrigeração, ao 1-MCP e à atmosfera modificada passiva.

\section{MATERIAL E MÉTODOS}

Os frutos foram colhidos com a coloração verde, na região da Serra da Bodoquena-MS, transportados em caixas plásticas até o Laboratório de Tecnologia de Alimentos da UFMS, e submetidos à seleção, com descarte dos frutos muito pequenos e com textura amolecida. Foram separados em dois grupos e ainda no mesmo dia um grupo recebeu aplicação de 1-MCP $700 \eta \mathrm{L} . \mathrm{L}^{-1}$. O gás 1-MCP foi gerado misturando-se $4 \mathrm{mg}$ de EthylBlock TM $(0,33 \%)$ da Rohm and Haas Química Ltda. e $3 \mathrm{~mL}$ de água destilada, injetada num frasco de vidro de $100 \mathrm{~mL}$, com tampa plástica. Após agitação, este foi destampado no interior de um isopor de $80 \mathrm{~L}$, lacrado com fita adesiva e mantido por 12 horas. Os frutos do grupo-controle também foram mantidos por 12 horas dentro de isopor de $80 \mathrm{~L}$ lacrado com fita adesiva.

Os tratamentos, compostos por 300 frutos cada, foram especificados a seguir: 1) Controle; 2) 1-MCP; 3) Atmosfera modificada passiva (AM); e 4) 1-MCP acondicionado sob atmosfera modificada passiva $(1 \mathrm{MCP}+\mathrm{AM})$.

O esquema fatorial utilizado foi 4 tratamentos x 2 temperaturas de armazenamento (ambiente e refrigerado) $\mathrm{x} 4$ períodos de tempo em dias.

A atmosfera modificada passiva foi obtida por meio de acondicionamento dos frutos em potes plásticos cobertos com PVC esticável com $13 \mu \mathrm{m}$ de espessura. 
Parte dos frutos foi armazenada em temperatura ambiente (A) $\left(28 \pm 4^{\circ} \mathrm{C}\right.$ e $50 \pm 10 \%$ UR), e a outra parte foi mantida sob refrigeração (R) $\left(11 \pm 0,5^{\circ} \mathrm{C}\right.$ e $80 \pm 5 \%$ UR) durante 10 dias, sendo em seguida armazenada em ambiente $\left(28 \pm 4^{\circ} \mathrm{C}\right.$ e 50 $\pm 10 \%$ UR) até a maioria dos frutos permanecer em boas condições para consumo.

Para acompanhar o comportamento das características dos frutos durante o armazenamento, foram realizadas análises iniciais e a cada 3 dias para os frutos armazenados em ambiente, e a cada 5 dias durante o armazenamento refrigerado.

A avaliação de perda de massa foi realizada por meio de pesagens das parcelas constituídas por 15 frutos, com 5 repetições por tratamento. Para as análises físico-químicas, foi obtida uma amostra composta por 25 frutos sem casca, homogeneizada, com 3 repetições por tratamento e análises em triplicata.

Os teores de sólidos solúveis e acidez titulável foram avaliados segundo metodologia do Instituto Adolfo Lutz (BRASIL, 2005) e a determinação do teor de ácido ascórbico foi por titulação com o reagente 2,6-diclorofenolindofenol (DCFI), de acordo com metodologia proposta pela Association of Official Analytical Chemists (1995).

As análises para os fenóis totais e atividade antioxidante foram realizadas no início e final do período de armazenamento, em triplicata. Foram preparados extratos aquosos com 10 gramas de polpa fresca e dissolvidos em metanol para uma concentração de sólidos de $3 \mathrm{mg} \cdot \mathrm{mL}^{-1}$, seguindo metodologia descrita em Roesler et al. (2007). $\mathrm{Na}$ determinação dos fenóis totais, os extratos metanólicos foram submetidos à reação colorimétrica descrita por Swain e Hills (1959) a $760 \mathrm{~nm}$, onde os compostos fenólicos presentes na amostra reduzem o reagente de Folin-Ciocalteau, formando um complexo azul de coloração intensa. A curva-padrão foi preparada com ácido gálico, e os resultados dos extratos expressos como equivalentes de ácido gálico (EAG) em mg de ácido gálico por $100 \mathrm{~g}$ de polpa fresca.

A capacidade antioxidante dos frutos foi mensurada por meio do método de sequestro de radicais livres DPPH (2,2-difenil-1-picril-hidrazil), em espectrofotômetro a $517 \mathrm{~nm}$ (ROESLER et al., 2007), e expressa como percentual de inibição de oxidação do radical, sendo o valor de IC50 definido como a concentração final do extrato requerido para decrescer a concentração inicial de DDPH em 50\%.

$\mathrm{Na}$ análise sensorial, foi avaliado o atributo aparência geral, por meio de teste visual, utilizando escala hedônica de 9 pontos, variando de gostei muitíssimo (valor 9) a desgostei muitíssimo (valor 1), com 35 julgadores não treinados, a fim de indicar a preferência entre os tratamentos, conforme descrito por Monteiro (2005). A avaliação foi realizada após 6 dias nos frutos armazenados em ambiente e após 16 dias nos armazenados em refrigeração.

Os dados foram submetidos à análise de variância (ANOVA) e, para comparação das médias, foi utilizado o teste de Tuckey, em nível de 5\% de probabilidade, com auxílio do programa estatístico BioEstat 5.0 (AYRES et al., 2007).

\section{RESULTADOS E DISCUSSÃO}

A aplicação das tecnologias pós-colheita, 1-MCP, atmosfera modificada e refrigeração interferiu estatisticamente na vida útil, perda de massa e teor de acidez titulável de guaviras. Houve interação entre os tratamentos utilizados e o período de armazenamento $(p<0,05)$ somente em relação à perda de massa dos frutos.

$\mathrm{O}$ uso de refrigeração a $11^{\circ} \mathrm{C}$ durante 10 dias prolongou a vida útil dos frutos-controle de 6 para 13 dias. A aplicação de 1-MCP e/ou atmosfera modificada passiva aumentaram em 3 dias a vida útil dos frutos, independentemente do armazenamento em ambiente ou refrigerado. A interferência positiva da refrigeração foi relatada por Morgado et al. (2010) em conservação pós-colheita de goiaba 'Kumagai', pertencente à mesma família da guavira, com maior vida útil quando armazenadas a $10^{\circ} \mathrm{C}$.

Resultados semelhantes quanto à eficiência do 1-MCP em retardar o amadurecimento de frutos foram relatados por Amarante et al. (2008), em goiaba serrana (500 $\eta L . L^{-1}$ por 8 horas) e por Cerqueira et al. (2009), em goiabas 'Kumagai' (300 a $900 \eta \mathrm{L} . \mathrm{L}^{-1}$ por 3 horas). Beneficio superior foi obtido com o uso de 1-MCP (1.000 $\eta \mathrm{L} \cdot \mathrm{L}^{-1}$ por 12 horas $) \mathrm{e}$ armazenamento em $10^{\circ} \mathrm{C}$, dobrando a vida útil de frutos de jambo (PLAINSIRICHAI et al., 2010).

A perda de massa dos frutos após 9 dias completos de armazenamento em temperatura ambiente foi elevada no Controle e nos que receberam 1 -MCP $(24,62 \%$ e $26,30 \%$, respectivamente), os quais diferiram dos demais tratamentos $(\mathrm{p} \leq 0,05 \%)$, e reduzida com a atmosfera modificada passiva e quando associada ao uso do 1-MCP, com valores finais de $12,86 \%$ e $12,68 \%$ de perda de massa dos frutos, respectivamente (Tabela 1, Figura 1-A). Resultado concordante para armazenamento em temperatura ambiente foi obtido por Cerqueira et al. (2009), onde o uso de 1-MCP não alterou a perda de massa em goiabas 'Kumagai', e por Batista et al. (2009), com valores de perda de massa inferiores 
utilizando a associação de 1-MCP e a atmosfera modificada por uso de ceras, em goiabas 'Paluma.'

O uso da refrigeração reduziu significativamente as perdas de massa dos frutos (Tabela 1, Figura 1-R). O Controle (R) e os tratados com 1-MCP (R), após 16 dias completos, apresentaram $21,13 \%$ e $21,05 \%$, respectivamente, e as menores perdas de massa acumulada foram obtidas com o uso da atmosfera modificada passiva: $8,20 \%$ AM (R) e 7,15\% 1-MCP+AM (R), sem diferenças significativas entre si.

O teor de acidez titulável inicial dos frutos foi de $0,33 \mathrm{~g}$ de ácido cítrico. $100 \mathrm{~g}^{-1}$ de polpa, ocorrendo pequenas variações durante $\mathrm{o}$ armazenamento com elevação dos valores finais nos frutos refrigerados (Tabela 1, Figura 2). No final do período de armazenamento em temperatura ambiente, os frutos-controle atingiram $0,42 \mathrm{~g} .100 \mathrm{~g} \mathrm{~g}^{-1}$, e os demais tratamentos variaram entre 0,32 e 0,38 g. $100 \mathrm{~g} \mathrm{~g}^{-1}$, enquanto os frutos armazenados refrigerados sob atmosfera modificada passiva atingiram os maiores valores, 0,46 g. $100 \mathrm{~g} \mathrm{~g}^{-1}$ de polpa, diferindo estatisticamente dos demais. Freitas et al. (2008) encontraram valores médios de acidez titulável de 0,29 g. $100 \mathrm{~g}^{-1}$ em guaviras Campomanesia sp. in natura, enquanto estudando frutos maduros, espécie C. xanthocarpa (Berg), Santos et al. (2009) relataram valores de $0,48 \mathrm{~g}$ ácido cítrico. $100 \mathrm{~g}^{-1} \mathrm{de}$ polpa.

Silva et al. (2001) consideraram que o amadurecimento reduziu a acidez presente nos frutos de guavira, o que não ocorreu no presente trabalho. De acordo com Chitarra e Chitarra (2005), geralmente, o teor de ácidos orgânicos diminui com a maturação dos frutos em decorrência do seu uso como substrato respiratório ou de sua conversão em açúcares, entretanto os compostos fenólicos também apresentam caráter acídico, podendo de certa forma contribuir para a acidez. Segundo Alves et al. (2000), um aumento da acidez deve-se à liberação dos ácidos galacturônicos que aumentam com o amadurecimento do fruto pela ação das enzimas pectinametilesterase e poligalacturonase, o que é condizente com o alto teor de pectina detectado em guavira por Santos et al. (2009).

Teores mais altos de acidez titulável com o uso de 1-MCP foram descritos para frutos climatéricos, como caqui (BRACKMANN et al., 2003), mangas 'Kent', (DOLLHOJO et al., 2009) e mangaba (CAMPOS et al., 2011), assim como para ameixas armazenadas sob atmosfera modificada e refrigeração (STEFFENS et al., 2009), possivelmente devido à diminuição do metabolismo respiratório e, consequentemente, menor consumo dos ácidos orgânicos.

Os teores médios de sólidos solúveis (SS) variaram durante o período de armazenamento (Figura 2) e entre os tratamentos, com valores finais entre 12,39 e $14,77^{\circ}$ Brix, os quais não diferiram estatisticamente entre si e com o valor inicial de 13,02 ${ }^{\circ}$ Brix (Tabela 1). Valores semelhantes foram relatados para guavira in natura $13,2^{\circ}$ Brix (SILVA et al., 2001); $11,00 \pm 1,41^{\circ}$ Brix (FREITAS et al., 2008) e 12,25 \pm $0,50{ }^{\circ}$ Brix (SANTOS et al., 2009). Assim como no presente trabalho, os teores de SS não foram sensíveis ao tratamento com 1-MCP, em goiabas serranas (AMARANTE et al., 2008), em goiaba 'Paluma' com associação a ceras (BATISTA et al., 2009).

$\mathrm{O}$ teor de vitamina $\mathrm{C}$ nos frutos frescos foi de $138,39 \mathrm{mg}$ de ácido ascórbico. $100 \mathrm{~g}^{-1} \mathrm{de}$ polpa, apresentando variações durante o período de armazenamento (Figura 2). Não houve diferenças estatísticas entre os valores finais dos frutos submetidos aos diferentes tratamentos e condições de armazenamento, variando entre $123,76 \mathrm{mg} .100 \mathrm{~g}^{-1}$ (1-MCP+AM) em ambiente e 147,06 g.100 g-1 (AM) sob refrigeração (Tabela 1). Silva et al. (2001) encontraram valores baixos de vitamina C em guaviras maduras, $21,0 \mathrm{mg} .100 \mathrm{~g}^{-1}$ de polpa, enquanto Vallilo et al. (2006) encontraram valores elevados (234 $\pm 2 \mathrm{mg} .100 \mathrm{~g} \mathrm{~g}^{-1}$ de polpa) em guavira C. adamantinum, assim como Santos et al. (2009) em C. xanthocarpa (Berg) $\left(233,56 \pm 11 \mathrm{mg} .100 \mathrm{~g}^{-1}\right)$, demonstrando variações entre espécies e regiões de ocorrência.

Os teores de ácido ascórbico não diferiram significativamente entre frutos de goiaba 'Paluma' com aplicação de 1-MCP e sua associação com ceras, entretanto ocorreu redução nos valores de ácido ascórbico no decorrer do tempo de armazenamento relatado por Batista et al. (2009), a qual não foi observada no presente trabalho.

A polpa fresca de guavira apresentou teor inicial de fenóis totais equivalente a $249,49 \mathrm{mg}$ EAG.100 g ${ }^{-1}$. Frutos armazenados em temperatura ambiente mostraram conteúdo final de compostos fenólicos variando entre 208,12 mg EAG.100 $\mathrm{g}^{-1}$ (AM) e 263,88 mg EAG.100 g-1 de polpa (1-MCP), e entre 229,41 mg EAG.100g-1 (AM) e 255,99 mg EAG.100 $\mathrm{g}^{-1}$ de polpa (Controle) quando em armazenamento refrigerado (Figura 3).

Valores semelhantes de polifenóis foram encontrados em pequenos frutos pertencentes ao gênero Rubus, Ribes e Aronia, entre 140,6 e 888,5 mg.100 g-1 (BENVENUTI et al., 2004), outros frutos da família Myrtaceae, como jabuticaba, jambolão e uvaia, com 440; 187 e 127 mg EAG.100 g-1, respectivamente (RUFINO et al., 2010), em guavira, 
espécie C. xanthocarpa (Berg), 161,6 \pm 27 mg.100 $\mathrm{g}^{-1}$ (SANTOS et al., 2009), em polpa de goiaba congelada 124 mg EAG.100 g ${ }^{-1}$ (HASSIMOTO et al., 2005). Rufino et al. (2010) sugeriram uma classificação dos teores de fenóis totais como baixo $\left(<100 \mathrm{mg}\right.$ EAG $\left.100 \mathrm{~g}^{-1}\right)$, médio (100-500 mg EAG $\left.100 \mathrm{~g}^{-1}\right)$ e alto ( $>500 \mathrm{mg}$ EAG $\left.100 \mathrm{~g}^{-1}\right)$ na matéria fresca; dessa forma, a guavira pode ser considerada como fruto de médio teor de fenóis totais.

A atividade antioxidante dos frutos, expressa como concentração final do extrato necessária para inibir a oxidação do radical DPPH em 50\% $\left(\mathrm{IC}_{50}\right)$, foi inicialmente de $140,57 \mu \mathrm{g} \cdot \mathrm{mL}^{-1}$, com valores finais entre 76,87 (AM) e 93,48 $\mu \mathrm{g} \cdot \mathrm{mL}^{-1}$ (Controle) em frutos armazenados em ambiente e 101,05 (Controle) e 145,71 $\mu \mathrm{g} \cdot \mathrm{mL}^{-1}(1-\mathrm{MCP}+\mathrm{AM})$ em frutos armazenados sob refrigeração (Figura 3). Quanto menor o valor de $\mathrm{IC}_{50}$ maior é a atividade antioxidante do extrato, portanto observou-se que, no caso da guavira, quanto mais madura maior a atividade antioxidante.

Valores semelhantes, entre 148,82 e 387,47 $\mu \mathrm{g} . \mathrm{mL}^{-1}$, foram encontrados em extratos etanólicos de outros frutos nativos como araticum, lobeira, pequi e cagaita (ROESLER et al., 2007). Iha et al. (2008) encontraram IC ${ }_{50}$ de $150 \mu \mathrm{g} . \mathrm{mL}^{-1}$ em extrato etanólico de goiaba, e Reynertson et al. (2008), estudando frutos de 14 espécies de Myrtaceae, relataram valores entre 19,4 $\mu \mathrm{g} \cdot \mathrm{mL}^{-1}$ (jabuticaba) a
$389 \mu \mathrm{g} \cdot \mathrm{mL}^{-1}$ (jambolão).

Aumento nos teores de vitamina $\mathrm{C}$, de polifenóis extraíveis totais e da atividade antioxidante foram relatados por Morgado et al. (2010) em goiabas "de vez", armazenadas a $10^{\circ} \mathrm{C}$. No presente trabalho, esses teores mantiveram-se próximos do valor inicial com o armazenamento refrigerado.

Para o atributo aparência geral dos frutos, os tratamentos aplicados apresentaram valores estatisticamente superiores aos controles ambiente e refrigerado, os quais obtiveram notas em torno do termo "desgostei moderadamente", de 2,91 e 3,26, respectivamente (Figura 4). A aplicação de 1-MCP apresentou melhor aparência geral dos frutos quando associada ao armazenamento refrigerado, 5,97 ("gostei ligeiramente"), diferindo estatisticamente do ambiente, 4,47 (entre "desgostei moderadamente" e "ligeiramente").

Os frutos tratados com 1-MCP+AM refrigerado apresentaram maior valor $(7,85)$, estando entre os termos "gostei moderadamente" e "gostei muito"; entretanto, não houve diferença estatística com o mesmo tratamento armazenado em ambiente $(6,91)$ e o AM ambiente $(6,82)$. A preferência dos julgadores correspondeu às menores perdas de massa, demonstrando a importância do uso de embalagens adequadas na manutenção da qualidade dos frutos.

TABELA 1 - Valores médios das análises físicas e químicas dos frutos de guavira submetidos à aplicação de 1-MCP 700 ๆL.L-1 (1-MCP), acondicionamento em atmosfera modificada passiva (AM), ou associação entre estes (1-MCP+AM), após armazenamento ambiente $\left(28 \pm 4^{\circ} \mathrm{C}\right.$ e $50 \pm$ $10 \%$ de UR) ou armazenamento refrigerado, durante 10 dias $\left(11 \pm 0,5^{\circ} \mathrm{C}\right.$ e $80 \pm 5 \%$ de UR), seguido de armazenamento ambiente.

\begin{tabular}{|c|c|c|c|c|c|}
\hline Tratamentos & $\begin{array}{l}\text { Vida útil } \\
\text { (dias) }\end{array}$ & $\begin{array}{c}\text { Perda de massa total } \\
(\%)\end{array}$ & $\begin{array}{l}\text { Sólidos Solúveis } \\
\qquad\left({ }^{\circ} \text { Brix }\right)\end{array}$ & $\begin{array}{c}\text { Acidez Titulável } \\
\left(\mathrm{g} \text { ác. cítrico. } 100 \mathrm{~g}^{-1}\right)\end{array}$ & $\begin{array}{c}\text { Vit } \mathrm{C} \\
\left(\mathrm{mg} \text { ác. ascórbico. } 100 \mathrm{~g}^{-1}\right)\end{array}$ \\
\hline$\frac{\text { Inicial }}{\text { Ambiente }}$ & - & - & $13,02 \pm 0,93 \mathrm{~ns}^{*}$ & $0,328 \pm 0,066 \mathrm{a}$ & $138,39 \pm 10,02 \mathrm{~ns}$ \\
\hline Controle & $6 a$ & $24,62 \pm 1,97 \mathrm{ab}$ & $13,15 \pm 1,23$ & $0,423 \pm 0,007 \mathrm{~d}$ & $127,34 \pm 18,28$ \\
\hline $1 \mathrm{Mcp}$ & $9 a b$ & $26,30 \pm 1,91 \mathrm{a}$ & $14,77 \pm 0,64$ & $0,375 \pm 0,017 \mathrm{c}$ & $143,22 \pm 11,71$ \\
\hline $\mathrm{AM}$ & $9 a b$ & $12,86 \pm 1,22 \mathrm{c}$ & $12,39 \pm 0,56$ & $0,348 \pm 0,001 \mathrm{~b}$ & $139,61 \pm 10,04$ \\
\hline $1 \mathrm{Mcp}+\mathrm{AM}$ & $9 \mathrm{ab}$ & $12,68 \pm 1,00 \mathrm{c}$ & $13,57 \pm 2,06$ & $0,320 \pm 0,009 \mathrm{a}$ & $123,76 \pm 2,81$ \\
\hline \multicolumn{6}{|l|}{$\underline{\text { Refrigerado }}$} \\
\hline Controle & $13 \mathrm{bc}$ & $21,13 \pm 1,28 \mathrm{~b}$ & $13,46 \pm 1,26$ & $0,394 \pm 0,009 \mathrm{c}$ & $141,06 \pm 8,13$ \\
\hline $1 \mathrm{Mcp}$ & $16 \mathrm{c}$ & $21,05 \pm 2,24 \mathrm{~b}$ & $13,46 \pm 1,76$ & $0,397 \pm 0,008 \mathrm{c}$ & $137,11 \pm 9,01$ \\
\hline $\mathrm{AM}$ & $16 \mathrm{c}$ & $8,20 \pm 0,70 \mathrm{~d}$ & $13,16 \pm 1,27$ & $0,456 \pm 0,002 \mathrm{e}$ & $147,38 \pm 2,92$ \\
\hline $1 \mathrm{Mcp}+\mathrm{AM}$ & $16 \mathrm{c}$ & $7,15 \pm 0,28 \mathrm{~d}$ & $12,53 \pm 0,78$ & $0,367 \pm 0,017 \mathrm{~b}$ & $125,16 \pm 4,18$ \\
\hline
\end{tabular}

*Médias com letras iguais não diferem significativamente entre si $(\mathrm{p} \leq 0,05)$. 
(A) Ambiente

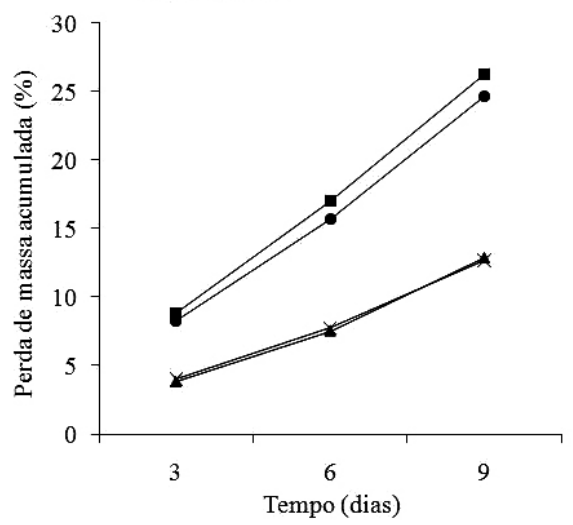

(R) Refrigerado

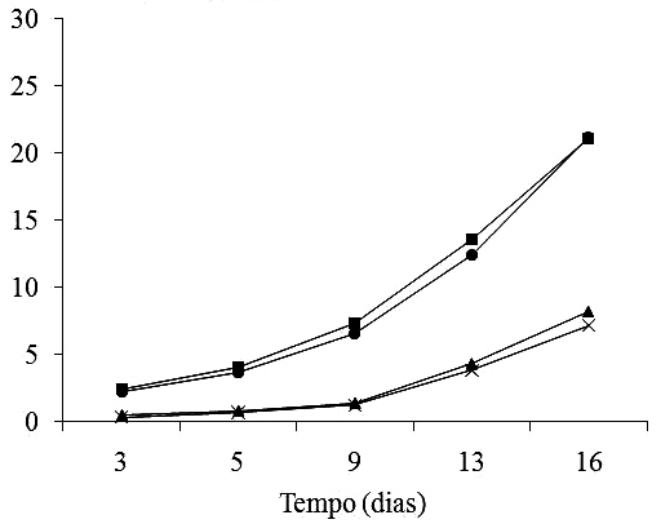

FIGURA 1 - Perda de massa acumulada de guaviras submetidas à aplicação de 1-MCP 700 ๆL.L-1 (1-MCP), acondicionadas em atmosfera modificada passiva (AM), ou associação entre estes (1-MCP+AM), durante armazenamento ambiente $\left(28 \pm 4^{\circ} \mathrm{C}\right.$ e $50 \pm 10 \%$ de UR) e armazenamento refrigerado, durante 10 dias $\left(11 \pm 0,5^{\circ} \mathrm{C}\right.$ e $80 \pm 5 \%$ de UR).

\section{- Controle $-1-\mathrm{MCP} \_\mathrm{AM} \star 1-\mathrm{MCP}+\mathrm{AM}$}
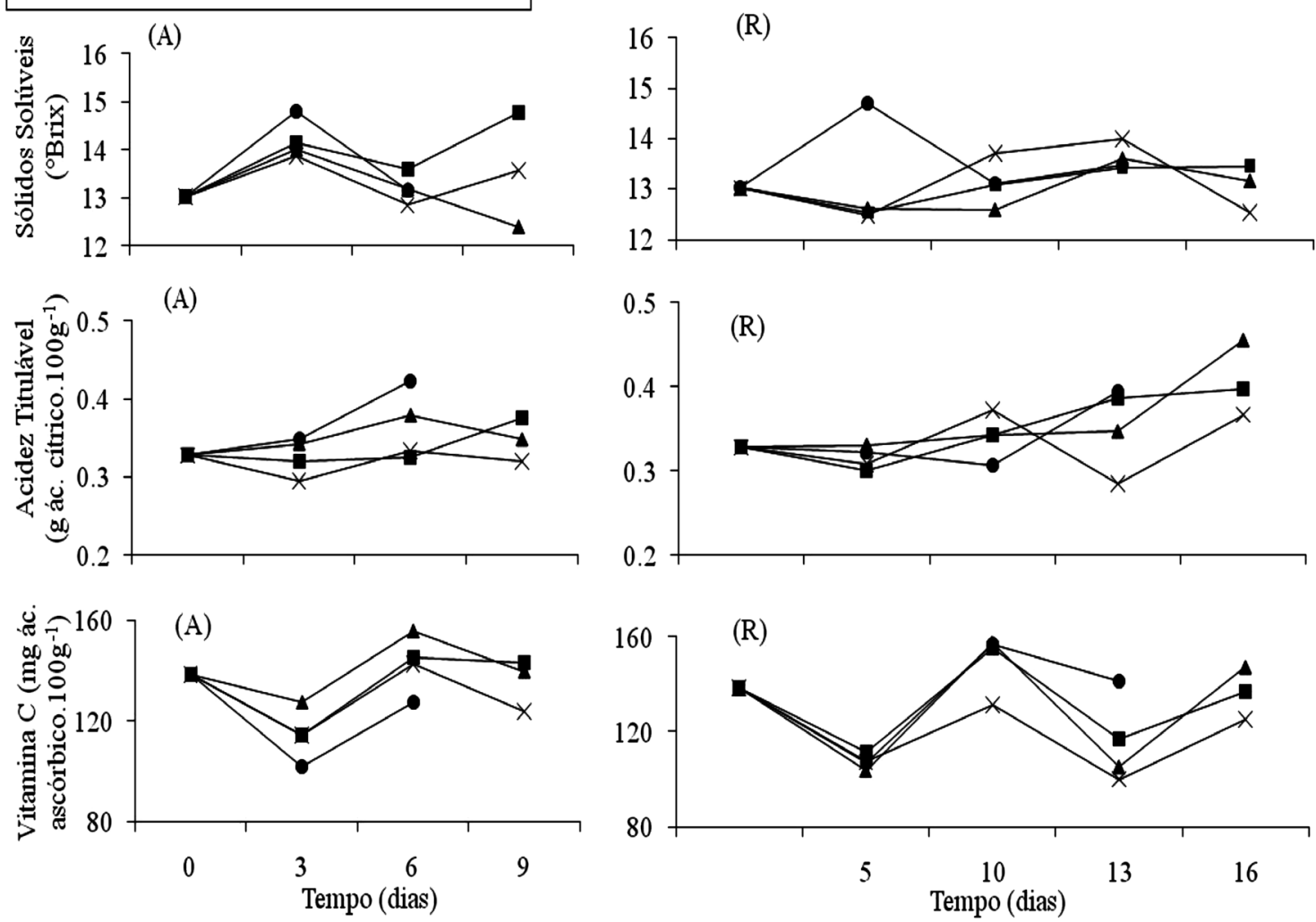

FIGURA 2 - Teores médios de sólidos solúveis, acidez titulável e vitamina $\mathrm{C}$ em guaviras submetidas à aplicação de 1-MCP $700 \eta \mathrm{L} . \mathrm{L}^{-1}$, à atmosfera modificada passiva (AM), à associação entre estes (1-MCP+AM), durante armazenamento ambiente (A) $\left(28 \pm 4^{\circ} \mathrm{C}\right.$ e $50 \pm 10 \%$ de UR) e armazenamento refrigerado (R), durante 10 dias $\left(11 \pm 0,5^{\circ} \mathrm{C}\right.$ e $80 \pm 5 \%$ de UR), posteriormente mantido em temperatura ambiente. 


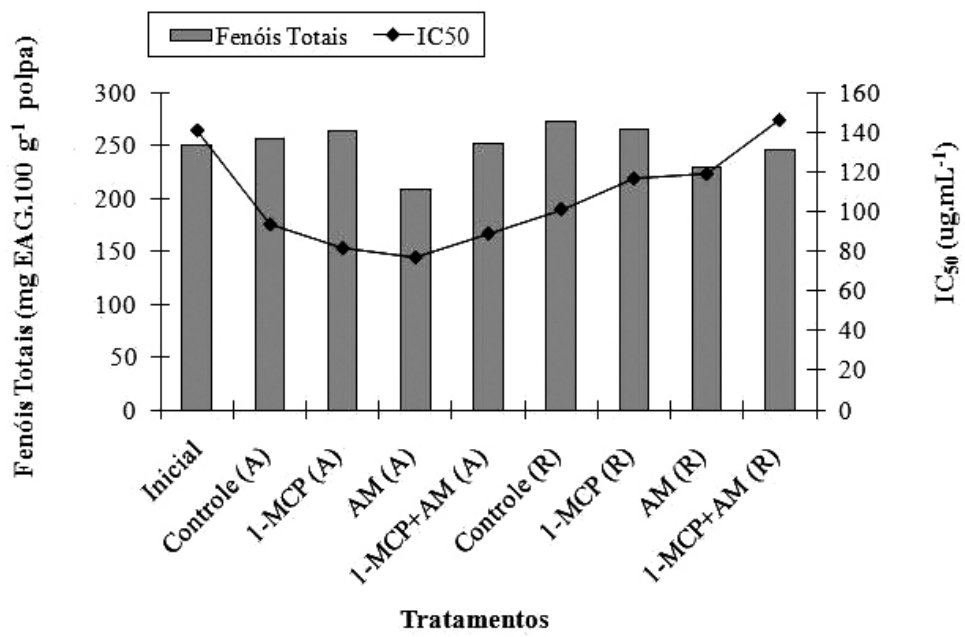

FIGURA 3 - Valores de fenóis totais e atividade antioxidante em guaviras submetidas à aplicação de 1-MCP $700 \eta \mathrm{L} . \mathrm{L}^{-1}$, à atmosfera modificada passiva (AM), à associação entre estes (1-MCP+AM), após seis dias de armazenamento ambiente (A) $\left(28 \pm 4^{\circ} \mathrm{C}\right.$ e $50 \pm 10 \%$ de UR) e após 13 dias de armazenamento $(\mathrm{R})\left(10\right.$ dias refrigerado $\left(11 \pm 0,5^{\circ} \mathrm{C}\right.$ e $80 \pm 5 \%$ de UR $)+3$ dias em ambiente $)$.

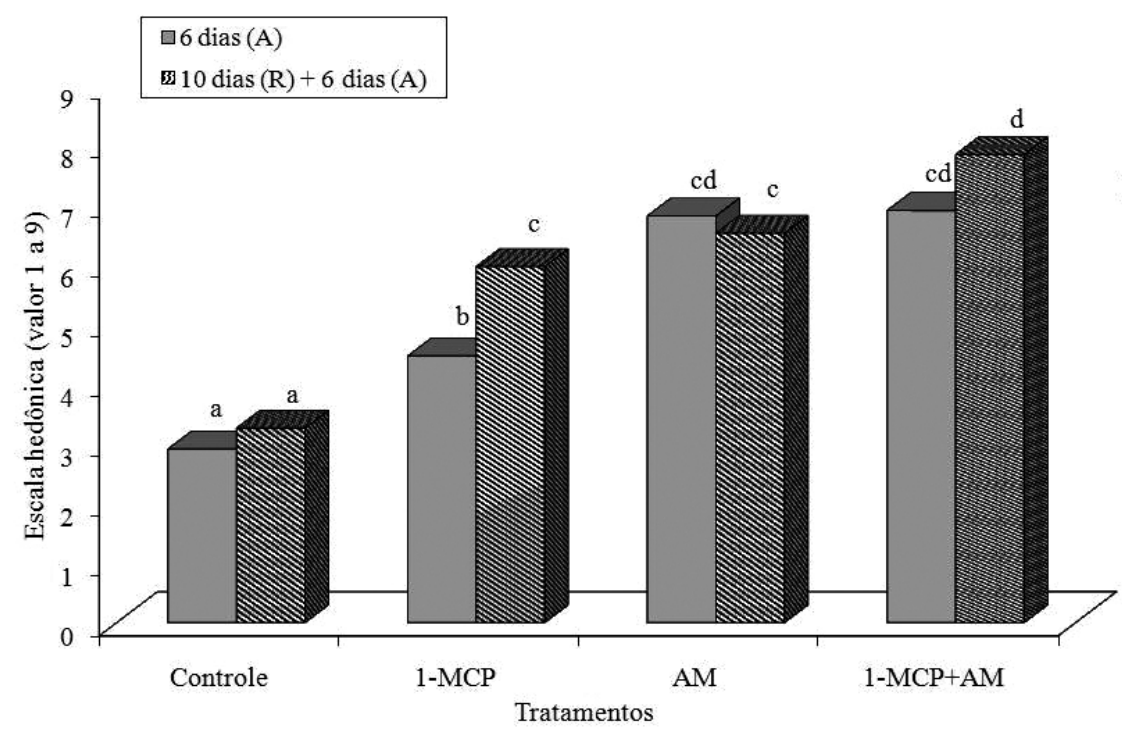

FIGURA 4 - Avaliação da aparência geral de guaviras submetidas à aplicação de 1-MCP 700 ๆL.L-1 , à atmosfera modificada passiva (AM), à associação entre estes (1-MCP+AM), após 6 dias de armazenamento ambiente (A) $\left(28 \pm 4^{\circ} \mathrm{C}\right.$ e $50 \pm 10 \%$ de UR) e armazenamento refrigerado (R) durante 10 dias $\left(11 \pm 0,5^{\circ} \mathrm{C}\right.$ e $80 \pm 5 \%$ de UR $)+6$ dias em ambiente. Valores finais seguidos de mesma letra não diferem entre si, pelo teste de Tuckey, a $5 \%$ de probabilidade. 


\section{CONCLUSÃO}

1-A conservação pós-colheita de guaviras é favorecida pelo armazenamento refrigerado a $11^{\circ} \mathrm{C}$, com maior vida útil e teores elevados de sólidos solúveis, acidez titulável, vitamina $\mathrm{C}$, fenóis totais e atividade antioxidante.

2-O uso de 1-MCP e de atmosfera modificada passiva prolongam a vida útil dos frutos tanto em armazenamento ambiente, quanto em refrigerado, com melhor aparência geral dos frutos, principalmente quando associados.

\section{AGRADECIMENTOS}

Os autores agradecem à Fundação de Pesquisa de Mato Grosso do Sul (Fundect) e ao Conselho Nacional de Desenvolvimento Científico e Tecnológico (CNPq), pelo apoio financeiro.

\section{REFERÊNCIAS}

ALVES, R. E.; FILGUEIRAS, H. A. C.; MOURA, C. F. H. Caracterização de frutas nativas da América latina. Jaboticabal: Funep, 2000. 66 p. (Série frutas nativas, 9).

AMARANTE, C. V. T. do; STEFFENS, C. A.; DUCROQUET, J. P. H. J.; SASSO, A. Qualidade de goiaba-serrana em resposta à temperatura de armazenamento e ao tratamento com 1-metilciclopropeno. Pesquisa Agropecuária Brasileira, Brasília, v. 43, n. 12, p. 1683-1689, 2008.

ASSOCIATION OF OFFICIAL ANALYTICAL CHEMISTS. ascorbic acid in vitamin preparations and juices. 2,6-dichloroindophenol titrimetric method. In: ASSOCIATION OF OFFICIAL ANALYTICAL CHEMISTS. Official methods of analysis of AOAC international. Washington: AOAC International, 1995. cap.45, p.16-17.

AYRES, M.; AYRES JR., M.; AYRES, D. L.; SANTOS, A. de A. S. Aplicações estatísticas nas áreas das ciências biomédicas: BioEstat 5.0. Belém, 2007. 324p.

BATISTA, P. F.; PEREIRA, M. de C.; SANTOS, A. E. O. dos; RIBEIRO, V. G.; ASSIS, J. S. Associação de 1-MCP com ceras de carnaúba na conservação de goiabas 'Paluma'. Revista Brasileira de Ciências Agrárias, Recife, v. 4, n. 1, p. 22-26, 2009.
BENVENUTI, S.; PELLATI, F.; MELEGARI, M.; BERTELLI, D. Polyphenols, Anthocyanins, Ascorbic Acid, and Radical Scavenging Activity of Rubus, Ribes, and Aronia. Jounal of Food Science, Chicago, v. 69, n. 3, p. 164-169, 2004.

BRASIL. Ministério da Saúde. Agência Nacional de Vigilância Sanitária. Métodos físico-químicos para análises de alimentos. Brasília: Ministério da Saúde, 2005. 1018p.

BRACKMANN, A.; FREITAS, S.T.; MELLO, A.M.; STEFFENS, C.A. Aplicação de 1-MCP em caqui 'Quioto' armazenado sob refrigeração e atmosfera controlada. Revista Brasileira de Fruticultura, Jaboticabal, v. 25, n. 1, p. 42-44, 2003.

CERQUEIRA, T. S.; JACOMINO, A. P.; SASAKI, F. F.; AMORIM, L. Controle do amadurecimento de goiabas 'Kumagai' tratadas com 1-metilciclopropeno. Revista Brasileira de Fruticultura, Jaboticabal, v. 31, n. 3, p. 687-692, 2009.

CAMPOS, R. P.; KNOCH, B.; HIANE, P. A.; RAMOS, M. I. L.; RAMOS FILHO, M. M. 1-MCP em mangabas armazenadas em temperatura ambiente e a $11^{\circ} \mathrm{C}$. Revista Brasileira de Fruticultura, Jaboticabal, v. 33, p. 206-212, 2011. Edição especial

CHITARRA, M. I. F.; CHITARRA, A. B. Póscolheita de frutos e hortaliças: fisiologia e manuseio. 2. ed. ver. e ampl. Lavras: UFLA, 2005. $785 \mathrm{p}$.

DOLLHOJO, E. T.; ABREU, C. M. P. DE; ASMAR, S. A.; HOJO, R. H.; CÔRREA, A. D.; VILAS BOAS, E. V. de B. Avaliação da qualidade de manga 'palmer' tratada com 1-metilciclopropeno e armazenada sob refrigeração e condição ambiente. Revista Brasileira de Fruticultura, Jaboticabal, v. 31, n. 1, p. 28-38. 2009

FREITAS, J. B.; CÂNDIDO, T. L. N.; SILVA, M R. Geleia de gabiroba: avaliação da aceitabilidade e características físicas e químicas. Pesquisa Agropecuária Tropical, Goiania, v. 38, n. 2, p. 87-94, 2008

HALLIWELL, B. Dietary poliphenols: good, bad or indifferent for your health. Cardiovascular Research, London, v. 73, p. 341-347, 2007. 
HASSIMOTO, N.M.A.; GENOVESE, M.I.; LAJOLO, F.M. Antioxidant activity of dietary fruits, vegetables, and commercial frozen fruit pulps. Jounal Agricultural and Food Chemistry, Washington, v. 53, n. 8, p. 2928-2935, 2005.

IHA, S. M.; MIGLIATO, K. F.; VELLOSA, J. C. R.; SACRAMENTO, L. V. S.; PIETRO, R. C. L. R.; ISAAC, V. L. B.; BRUNETTI, I. L.; CORRÊA, M.A.; SALGADO, H.R.N. Estudo fitoquímico de goiaba (Psidium guajava L.) com potencial antioxidante para o desenvolvimento de formulação fitocosmética. Revista Brasileira de Farmacognosia, João Pessoa, v. 18, n. 3, p.387-393, 2008.

KAUR, C.; KAPOOR, H. C. Anti-oxidant activity and total phenolic content of some Asian vegetables. International Journal of Food Science and Technology, Oxford, v.37, p.153-161, 2002.

MONTEIRO, A. R. G. Introdução à análise sensorial de alimentos. Maringá: Eduem, 2005. $46 \mathrm{p}$.

MORGADO, C. M. A.; DURIGAN, J. F.; LOPES, V. G.; SANTOS, L. O. Conservação pós-colheitade goiabas 'Kumagai': efeito da maturação e da temperatura de armazenamento. Revista Brasileira de Fruticultura, Jaboticabal, v. 32, n. 4, p. 10011008, 2010.

PLAINSIRICHAI, M.; TRINOK, U.; TURNER, W. D. 1-methylcyclopropene (1-MCP) reduces water loss and extends shelf life of fruits of Rose apple ( Syzygium jambos Alston) cv. Tabtim Chan. Fruits,Paris, v. 65, n. 133-140, 2010.

REYNERTSON, K. A.; YANG, H.; JIANG, B.; BASILE, M. J.; KENNELLY, E. J. Quantitative analysis of antiradical phenolic constituents from fourteen edible Myrtaceae fruits. Food Chemistry, Kidlington, v. 109, n. 4, p. 883-890, 2008.

RODRIGUES, V. E. G.; CARVALHO, D. A. Plantas medicinais no domínio dos cerrados. Lavras: Ed. UFLA, 2001. 180p.
ROESLER, R.; MALTA, L. G.; CARRASCO, L. C.; HOLANDA, R. B.; SOUZA, C. A. S.; PASTORE, G. M. Atividade antioxidante de frutas do cerrado. Ciência e Tecnologia de Alimentos, Campinas, v. 27, n. 1, p. 53-60, 2007.

RUFINO, M. S. M.; ALVES, R. E.; BRITO, E. S.; PÉREZ-JIMÉNEZ, J.; SAURA-CALIXTO, F.; MANCINI-FILHO, J. Bioactive compounds and antioxidant capacities of 18 non-traditional tropical fruits from Brazil. Food Chemistry, Kidlington, v. 121, p. 996-1002, 2010.

SANTOS, M. da S.; CARNEIRO, P.I. B.; WOSIACKI, G.; PETROWICZ, C. L. de O.; CARNEIRO, E. B. B. Caracterização físico-química, extração e análise de pectinas de frutos de Campomanesia Xanthocarpa B. (Gabiroba). Semina: Ciências Agrárias, Londrina, v. 30, n. 1, p. 101-106, 2009.

SILVA, D. B. da; SILVA, J. A. da; JUNQUEIRA, N. T. V.; ANDRADE, L. R. M. Frutos do cerrado. Brasília: Embrapa Informação Tecnológica, 2001. $178 \mathrm{p}$.

SILVA, M. R.; LACERDA, D. B. C. L.; SANTOS, G. G.; MARTINS, D. M. DE O. Caracterização química de frutos nativos do cerrado. Ciência Rural, Santa Maria, v. 38, n. 6, p. 1790-1793, 2008.

STEFFENS, C.A.; AMARANTE, C.V.T.; ALVES, E.O.; TANAKA, H.; BRACKMANN, A.; BOTH, V. Armazenamento de ameixas 'Laetitia' em atmosfera modificada. Ciência Rural, Santa Maria, v. 39, n. 9, p. 2439-2444, 2009.

SWAIN, T.; HILLS, W. E. The phenolics constituents of Prumus domestica: the quantitative analysis of phenolic constituents. Journal of the Science of Food and Agriculture, London, v. 10, n. 1, p. 6368, 1959.

VALLILO, M. I.; LAMARDO, L. C. A.; GABERLOTTI, M.; OLIVEIRA, E. de; MORENO, P. R. H. Composição química dos frutos de Campomanesia adamantium (Cambessédes) O.Berg. Ciência e Tecnologia de Alimentos, Campinas, v. 26, n. 4, p. 805-810, 2006. 